$\triangle$ Genome Biology

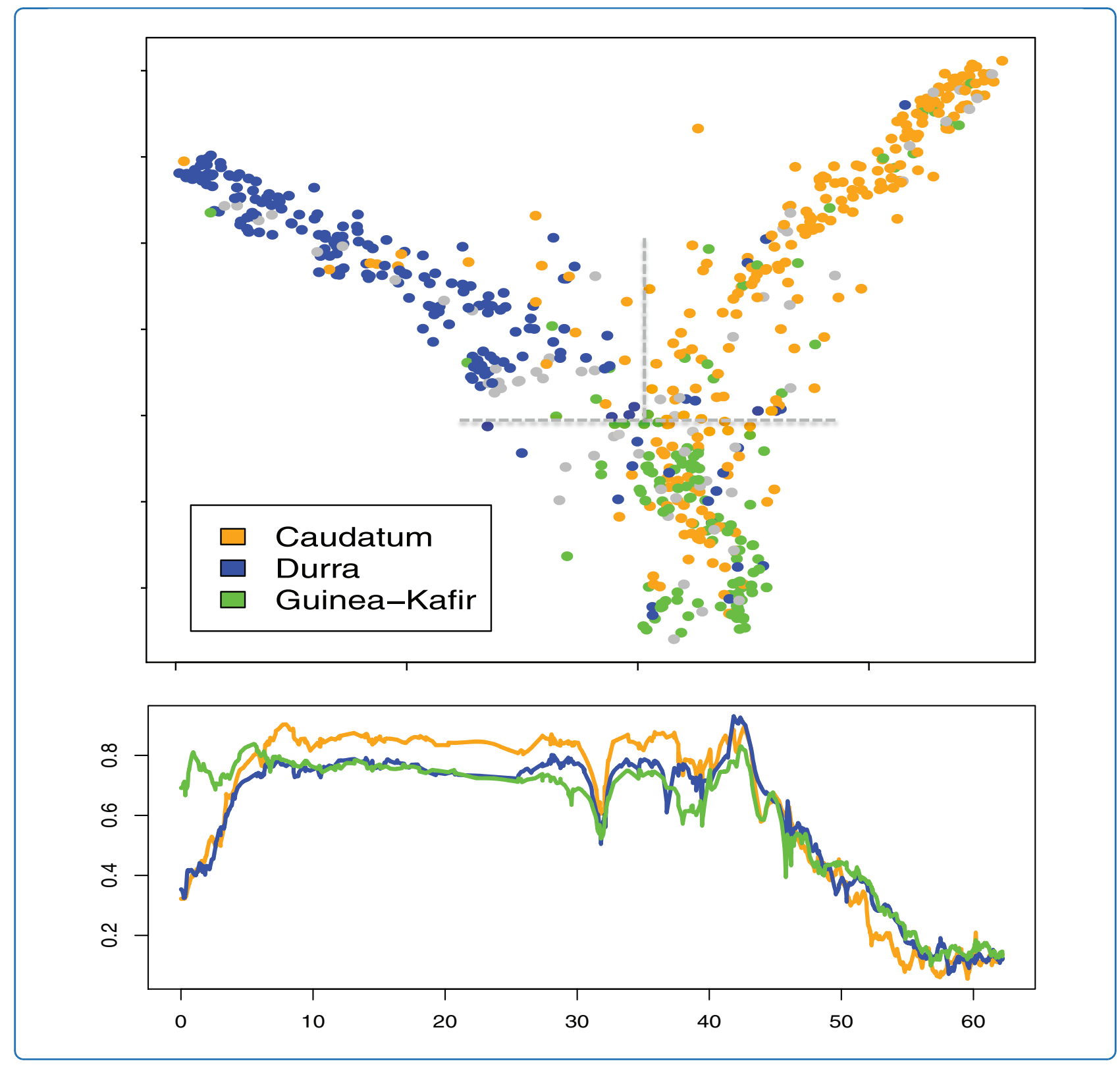

\title{
Retrospective genomic analysis of sorghum adaptation to temperate-zone grain production
}

Thurber et al. 


\title{
Retrospective genomic analysis of sorghum adaptation to temperate-zone grain production
}

\author{
Carrie S Thurber ${ }^{1}$, Justin M Ma ${ }^{1}$, Race H Higgins ${ }^{1,2}$ and Patrick J Brown ${ }^{1,2^{*}}$
}

\begin{abstract}
Background: Sorghum is a tropical $C_{4}$ cereal that recently adapted to temperate latitudes and mechanized grain harvest through selection for dwarfism and photoperiod-insensitivity. Quantitative trait loci for these traits have been introgressed from a dwarf temperate donor into hundreds of diverse sorghum landraces to yield the Sorghum Conversion lines. Here, we report the first comprehensive genomic analysis of the molecular changes underlying this adaptation.

Results: We apply genotyping-by-sequencing to 1,160 Sorghum Conversion lines and their exotic progenitors, and map donor introgressions in each Sorghum Conversion line. Many Sorghum Conversion lines carry unexpected haplotypes not found in either presumed parent. Genome-wide mapping of introgression frequencies reveals three genomic regions necessary for temperate adaptation across all Sorghum Conversion lines, containing the Dw1, Dw2, and Dw3 loci on chromosomes 9, 6, and 7 respectively. Association mapping of plant height and flowering time in Sorghum Conversion lines detects significant associations in the Dw1 but not the Dw2 or Dw3 regions. Subpopulation-specific introgression mapping suggests that chromosome 6 contains at least four loci required for temperate adaptation in different sorghum genetic backgrounds. The Dw1 region fractionates into separate quantitative trait loci for plant height and flowering time.

Conclusions: Generating Sorghum Conversion lines has been accompanied by substantial unintended gene flow. Sorghum adaptation to temperate-zone grain production involves a small number of genomic regions, each containing multiple linked loci for plant height and flowering time. Further characterization of these loci will accelerate the adaptation of sorghum and related grasses to new production systems for food and fuel.
\end{abstract}

Keywords: Genotyping-by-sequencing, introgression, photoperiod, flowering time, dwarfism

\section{Background}

Cereals have been selected by humans for thousands of years, first during their domestication from wild grasses and subsequently for increased yield, uniformity, and adaptation to new environments and management practices [1-3]. Specific molecular pathways have recently proven useful for cereal adaptation to modern, high-input agriculture. For example, the Green Revolution exploited allelic variation in the gibberellin pathway in wheat and rice to produce semi-dwarf cultivars with increased harvest index and improved resistance to lodging [4-7]. Similar phenotypic changes occurred during the creation of dwarf grain sorghum suitable for mechanized harvest

\footnotetext{
* Correspondence: pjb34@illinois.edu

'Energy Biosciences Institute, University of Illinois, Urbana, IL, USA

Full list of author information is available at the end of the article
}

at temperate latitudes. Understanding the genetic control of these changes is critical for the efficient transfer of useful alleles, both between tropical and temperate growing regions and between breeding programs for different end uses.

Sorghum is the fifth most important cereal crop worldwide [8] and is widely grown in temperate regions, but was domesticated in the African tropics [9]. Temperate adaptation for grain production in sorghum requires photoperiod-insensitivity, for early maturity, and dwarfism, both of which involve at least four major loci [10]. Of the major maturity loci (Ma1-Ma6), Ma1 has been identified as PRR37 [11] and Ma3 as Phytochrome B [12]. Of the major dwarfing loci (Dw1-Dw4), Dw3 has been identified as PGP1/PGP19, an auxin transporter orthologous to maize brachytic2 [13]. $D w 2$ and $D w 1$ are

\section{() Biomed Central}

(c) 2013 Thurber et al.; licensee BioMed Central Ltd. This is an open access article distributed under the terms of the Creative Commons Attribution License (http://creativecommons.org/licenses/by/2.0), which permits unrestricted use, distribution, and reproduction in any medium, provided the original work is properly cited. 
uncloned, with the former closely-linked to Ma1 [14] and the latter mapping to chromosome $9[15,16]$.

The oligogenic control of these important agronomic traits in sorghum was exploited through a backcross breeding scheme known as the Sorghum Conversion Program (SCP) [17]. Mutations for photoperiod-sensitivity and dwarfism had previously arisen spontaneously in temperate regions of Africa, Asia, and the southern US, and were already being used for grain sorghum production. However, the genetic base of US grain sorghum remained very narrow. During the SCP, genomic regions conferring early maturity and dwarfing were introgressed from an elite donor into approximately 800 exotic sorghum accessions representing the breadth of genetic diversity in sorghum. The resulting SC lines are closely related to their Exotic Progenitor (EP) lines, but differ dramatically in plant height and flowering time due to the presence of donor introgressions (Figure 1A). The elite donor, BTx406, carries recessive alleles for photoperiod-insensitivity and dwarfism at $M a 1$ and $D w 1-D w 3$, respectively [17], so these loci are expected to show a high frequency of donor introgression in SC lines. Klein et al. [14] previously mapped introgressions on chromosome 6 in a subset of SC lines and showed that several of them contain vast introgressed tracts around the linked Ma1-Dw2 loci. However, the genetic architecture of temperate adaptation in the SC lines (the number and linkage of loci as well as their frequencies in different subpopulations) has not been systematically studied on a genome-wide basis. This information can be used both to identify the underlying targets of the SCP and to help guide more efficient, marker-directed conversion of exotic sorghums to temperate-adapted varieties.

In this study, we use genotyping-by-sequencing (GBS) $[18,19]$ to generate genome-wide single nucleotide polymorphism (SNP) data for 580 pairs of EP and SC lines, for a total of 1,160 sorghum inbreds. We then employ a novel introgression mapping approach to identify loci required for temperate adaptation, and validate our results using both phenotype-genotype association and population differentiation $\left(\mathrm{F}_{\mathrm{st}}\right)$ analyses.

\section{Results and discussion}

\section{Genotyping-by-sequencing of SC lines and their exotic progenitors}

To map elite donor introgressions in SC lines, we genotyped 580 pairs of $S C$ and their corresponding EP lines (Additional File 1) at 54,034 SNPs using GBS. Briefly, we constructed reduced-representation DNA libraries using pairs of restriction enzymes [18], sequenced them in 96-plexes on the Illumina HiSeq, and processed the data using the TASSEL GBS pipeline [20]. We found that combining two separate double digests nearly doubled the number of SNPs called per sample (Additional File 2).
The full dataset contained $0.3 \%$ heterozygous genotypes. Partial imputation using the TASSEL GBS pipeline reduced the proportion of missing genotypes from $66 \%$ to $23 \%$.

Three different seed sources of the elite donor line, BTx406, were used to construct 28 different genomic libraries. Three of these libraries originating from a single seed source of BTx406 showed low concordance and were removed from subsequent analyses (Additional File 3). This low concordance was likely due to laboratory error as it was confined to libraries prepared on a single day. The remaining 25 libraries from the elite donor contained clear, homozygous majority calls for 53,037 SNPs. The elimination of approximately 7,000 SNPs in complete linkage disequilibrium with another SNP less than 64 basepairs (bp) away resulted in a dataset of 46,137 SNPs for calling introgressions.

Each trio of homozygous genotypes for a given SNP across a SC line, its corresponding EP line, and the elite donor has four possible outcomes (Figure 1B), most common of which is a lack of polymorphism. Of the three remaining polymorphic combinations, shared genotypes between a SC line and its EP line provide evidence that introgression has not occurred, whereas shared genotypes between a SC line and the donor provide evidence that introgression has occurred. The fourth possibility is unexpected: a SC line has a genotype not found in either of its parents. Unexpected genotypes could result from laboratory error (mix-up or cross-contamination of seed or DNA samples in our laboratory), historical error during the SCP (pollen contamination or error in pedigree records), or uncharacterized heterozygosity and/or genetic drift during the maintenance of the $\mathrm{EP}, \mathrm{SC}$, or donor lines. We used the proportion of unexpected genotypes as a quality-control filter to prune both markers and individuals. First, we discarded 75 markers with $>20 \%$ unexpected genotypes, of which 55 were on chromosome 6 and 44 were found between $30 \mathrm{Mb}$ and $43 \mathrm{Mb}$ on chromosome 6, a region that includes $M a 1$ and likely includes $D w 2$ [14]. A possible explanation for the high proportion of unexpected genotypes in this region is that certain sources of the elite donor BTx406 used during the SCP differed from our BTx406 consensus genotype in this region. In support of this hypothesis, we note that the seed source of BTx406 derived from Lubbock, TX, very close to where the SCP was carried out, is heterozygous for many of the markers on chromosome 6 that were discarded due to having $>20 \%$ unexpected genotypes. Second, we discarded 190 SC-EP pairs with $>10 \%$ unexpected genotypes. The distribution of unexpected genotypes in some SC lines is clustered (for example, SC1104; Additional File 4), suggesting that genomic segments from a temperate donor other than BTx406 were introgressed. In other SC lines the unexpected genotypes 


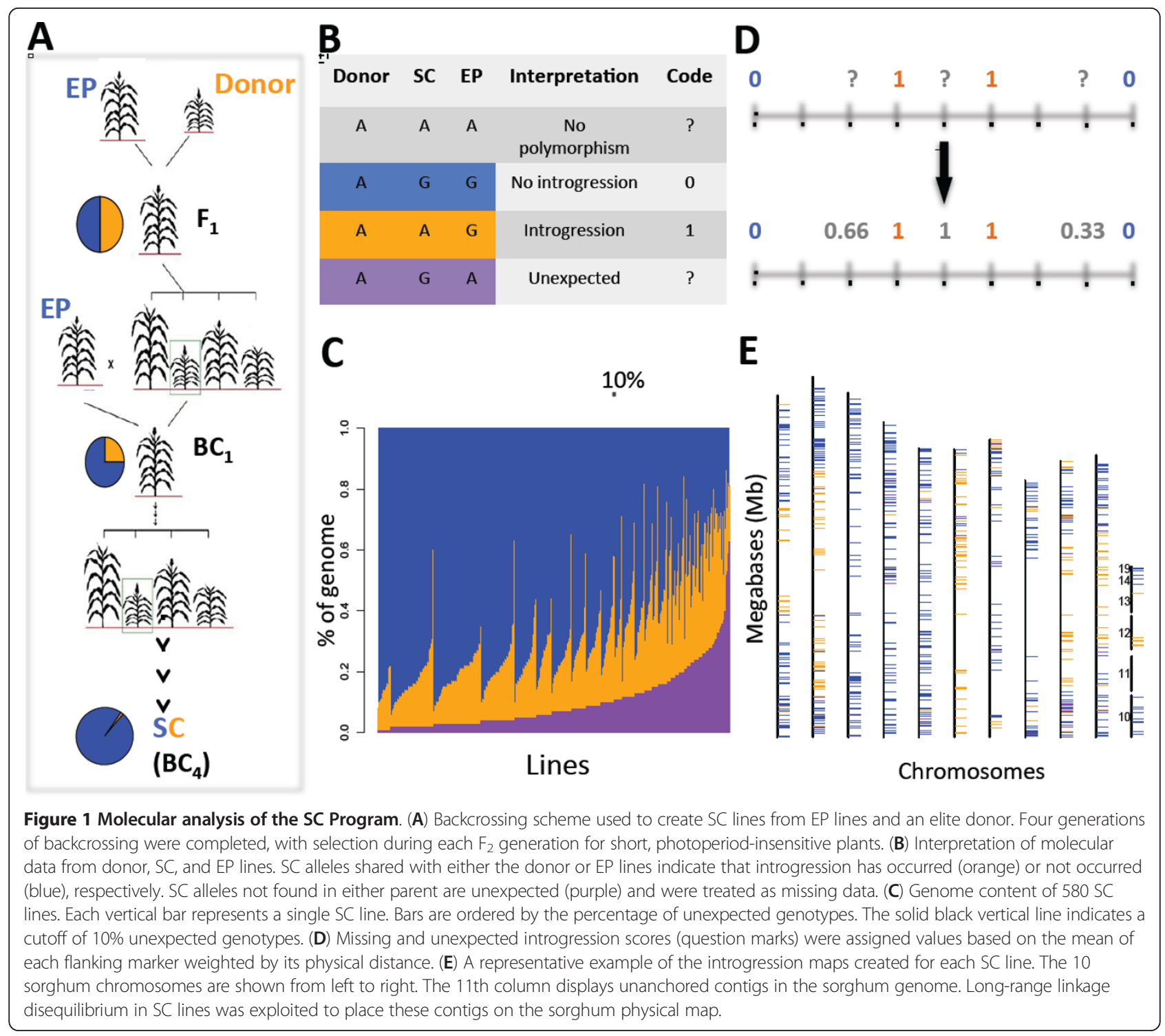

are scattered, suggesting that genetic drift may have occurred between the EP line that was used as a recurrent parent and the EP line that was genotyped. For the 16 SC-EP pairs that have $>33 \%$ unexpected genotypes, a clerical error of some kind - during transcription of pedigree records, seed packet labels, or DNA plates - is most likely. For the remainder of our analysis, we retained a set of 390 SC-EP pairs with $<10 \%$ unexpected genotypes (Figure 1C), genotyped at 46,062 markers (Additional File 5).

\section{Inferring elite donor introgressions in SC lines}

Introgression maps were generated for each SC line (Figure 1E; Additional File 4). The long-range linkage disequilibrium in the SC lines was exploited to map unanchored contigs in the sorghum genome (Additional File 6). After setting non-polymorphic and unexpected genotypes as missing, missing data were inferred using flanking markers (Figure 1D). Introgression frequency was then calculated for each marker as the proportion of the 390 SC lines carrying a BTx406 introgression. The theoretical expectation of introgression frequency after four backcrosses in the absence of selection is roughly $3 \%$. The standard deviation of this value in individual SC lines, in a species with 10 chromosomes and a map length of roughly 16 Morgans, is also roughly $3 \%$ [21], so that the introgression frequency in a sample of 390 SC lines is expected to range from $2 \%$ to $4 \%$ in the absence of selection. Because our dataset contains a substantial proportion of missing data, introgressions that are very small and very rare may be missed entirely. However, we find that every chromosome contains regions with introgression frequencies $>4 \%$, indicating linkage to a target of selection during the SCP. 
Three genomic regions are associated with temperate adaptation in sorghum

Three regions of the sorghum genome show pronounced peaks in introgression frequency in the SC lines (Figure 2; top panel), suggesting that these regions are nearly indispensable for adaptation to temperate grain production. We then used two methods to validate the introgression mapping results. First, we assessed functional variation for plant height and flowering time in SC lines by performing association mapping for these traits in the complete set of 580 genotyped SC lines (Figure 2; middle panel). EP lines were not included because most do not flower at temperate latitudes. Significant phenotypic associations were found in the $D w 1$

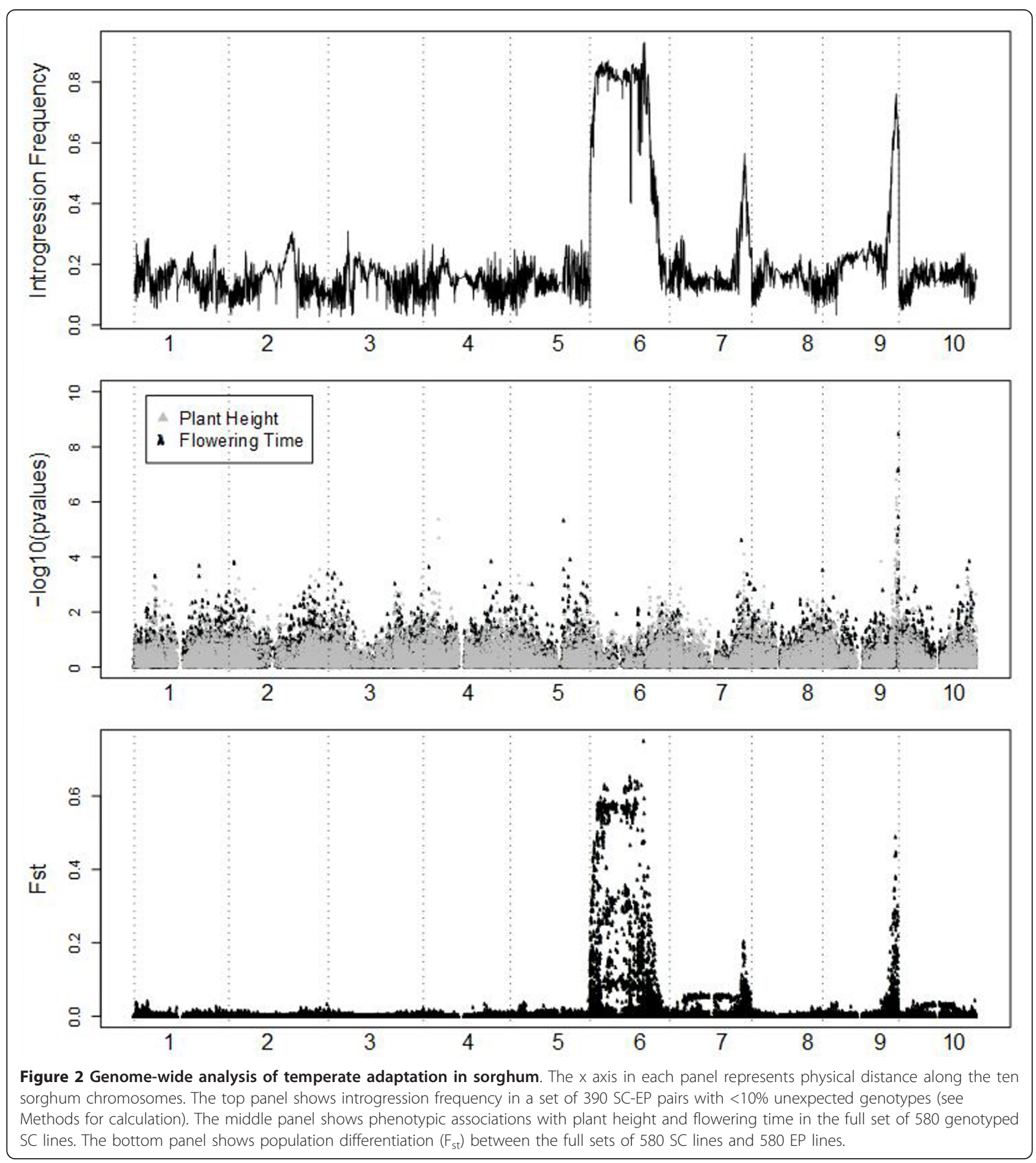


but not the $D w 2$ or $D w 3$ genomic regions. Second, to ensure that the introgression mapping results were not unduly affected by unexpected genotypes, we calculated $\mathrm{F}_{\mathrm{st}}$ between the complete sets of 580 genotyped SC lines and $580 \mathrm{EP}$ lines and found that regions of high $\mathrm{F}_{\mathrm{st}}$ mirror the regions of high introgression frequency almost exactly (Figure 2; bottom panel). Unlike introgression frequency, $F_{\text {st }}$ makes no assumptions about the pedigrees of the $\mathrm{SC}$ lines.

\section{The cloned Dw3 locus on chromosome 7 is tagged using three different methods}

Chromosome 7, which contains the known, cloned target $D w 3$ at $58.6 \mathrm{Mb}$, has a peak introgression frequency at $58.7 \mathrm{Mb}$, a peak $\mathrm{F}_{\mathrm{st}}$ at $58.6 \mathrm{Mb}$, and a peak plant height association at $58.2 \mathrm{Mb}$ that is not quite significant at $P<0.05$ following a Bonferonni correction (Figure 3). Since the causal mutation in $D w 3$ is a copy number variant $(\mathrm{CNV})$ that is unstable and may have arisen quite recently [13], our dataset may not contain linked SNPs in high linkage disequilibrium with the causal CNV. Several regions on either side of the $D w 3$ locus show local peaks in both introgression frequency and $\mathrm{F}_{\mathrm{st}}$, and co-localize with weak signals of flowering time association.

\section{The Dw1 region on chromosome 9 fractionates into linked QTL}

Chromosome 9, which contains the uncloned Dw1 locus, has a peak introgression frequency at $57.6 \mathrm{Mb}$, a peak $\mathrm{F}_{\mathrm{st}}$ at $57.4 \mathrm{Mb}$, and a peak plant height association at 57.5 $\mathrm{Mb}$, in close agreement with previous results (Figure 4) $[15,16]$. A separate cluster of SNPs in the Dw1 region associates with flowering time, with a peak at $59.6 \mathrm{Mb}$. The most significant SNPs for plant height and flowering time are not in significant linkage disequilibrium with each other $\left(r^{2}=0.15\right)$ and align with two distinct peaks in both introgression frequency and $\mathrm{F}_{\mathrm{st}}$, strongly suggesting that the $D w 1$ region contains separate loci for plant height and flowering time.

\section{Chromosome 6 retains little functional variation in SC lines}

Chromosome 6 displays a high introgression frequency and high $\mathrm{F}_{\mathrm{st}}$ across most of its length, even though the known targets on this chromosome are tightly linked: $M a 1$ at $40.3 \mathrm{Mb}$, and the uncloned $D w 2$ locus several $\mathrm{Mb}$ away (Figure 5). The peak introgression frequency and peak $\mathrm{F}_{\text {st }}$ on chromosome 6 apparently correspond to $D w 2$ and not Ma1 (Additional File 7), possibly because several independent recessive ma1 alleles already exist in the EP lines (R. Klein, personal communication). The choppiness of the introgression frequency between $30 \mathrm{Mb}$ and $43 \mathrm{Mb}$ correlates with a very high proportion of unexpected genotypes in this region, which could result from the existence of an additional, uncharacterized ma1- $d w 2$ haplotype in the elite donor. There are no significant phenotypic associations on chromosome 6 , suggesting that elite donor introgressions have removed most functional variation for plant height and flowering time on this chromosome in SC lines. Consistent with previous studies reporting a limited number of chromosome 6 haplotypes in SC lines [14,15], we observe the maintenance of high introgression frequency across most of the chromosome, which could be attributed to either a large number of targeted loci or to limited recombination between a few targets. Targets could result from direct selection for plant height and flowering time and/or indirect selection for vigor and adaptation to climatic and soil variation. Regardless of the biological explanation, decreased variation on chromosome 6 is a concern for temperate sorghum breeding. Of the 35 major-effect genes mapped in sorghum as of 2010 [22], seven map to chromosome 6 and four $\left(d, g c, P, R s_{1}\right)$ have been associated with resistance to biotic stresses including ergot, grain mold, and shoot fly [23-25]. Exotic alleles at these and other unidentified linked loci are at low frequency in $\mathrm{SC}$ lines, yet may be useful in future breeding efforts.

\section{Identification of subpopulation-specific introgression targets}

Sorghum is a crop with strong population sub-division and apparently multiple domestication events [26]. Therefore, we calculated introgression frequencies separately in three subpopulations corresponding to the caudatum (C; $n=137)$, durra (D; $n=131)$, and guinea/kafir (GK; $n=122)$ racial groups. Subpopulations were defined based on genetic criteria in the EP lines (see Methods), which closely match traditional morphological classification (Figure 6). Similar results were obtained when subpopulations are defined based on genetic criteria in the SC lines with or without the three major introgression regions included (Additional File 8). The significance of introgression frequency differences between subpopulations was assessed using permutation (see Methods). We identified multiple subpopulation-specific introgression targets on every chromosome (Additional File 9). Most dramatically, a target at approximately $1 \mathrm{Mb}$ on chromosome 6 is specific to the GK group. In addition to the linked Ma1-Dw2 loci and this GK-specific locus, the presence of at least one additional locus on chromosome 6 is necessary to explain the maintenance of high introgression frequency across the chromosome in SC lines of caudatum and durra origin. Introgression frequencies in regions linked to both $D w 1$ and $D w 3$ also vary significantly by subpopulation. Although differences in recombination between subpopulations could theoretically account for such differences, several of these regions also contain phenotypic associations with plant height and 


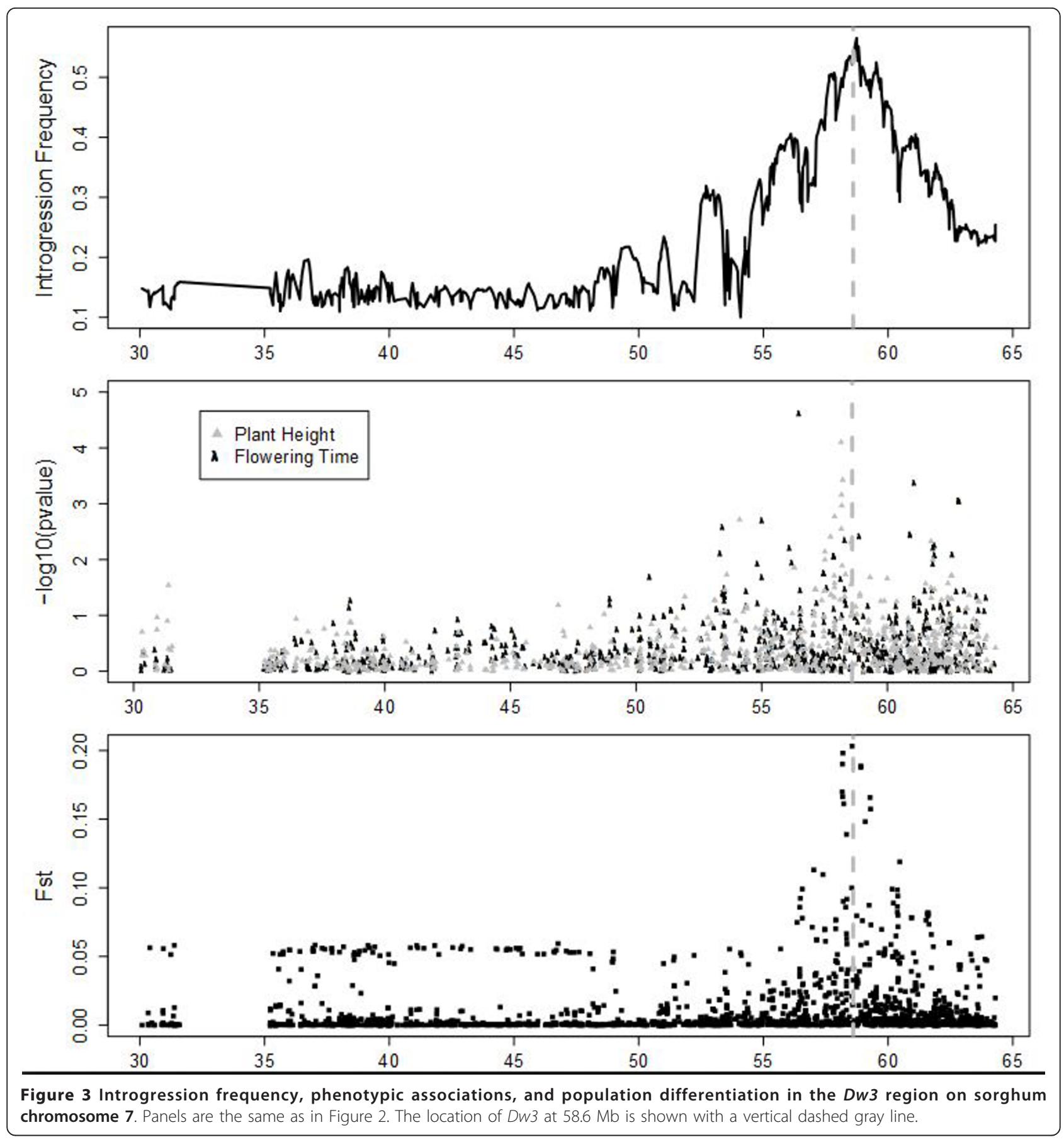

flowering time in SC lines, suggesting that they result from subpopulation-specific targets of the SCP. Similarly, a phenotypic association with flowering time at $41.9 \mathrm{Mb}$ on chromosome 5 overlaps with a GK-specific introgression peak (Figure 2, Additional Files 9 and 10). Additional subpopulation-specific targets in regions unlinked to $D w 1, D w 2$, and $D w 3$ that do not overlap with significant phenotypic associations could contain loci for other agronomic traits selected for during the conversion process, including disease resistance, lack of seed dormancy, and overall vigor under temperate conditions.

\section{Conclusions}

The molecular analysis of parents and progeny provides the opportunity for pedigree verification. Our results show that almost one-third of SC lines contain a substantial proportion of unexpected genotypes $(>10 \%$ of informative markers). We used three complementary 


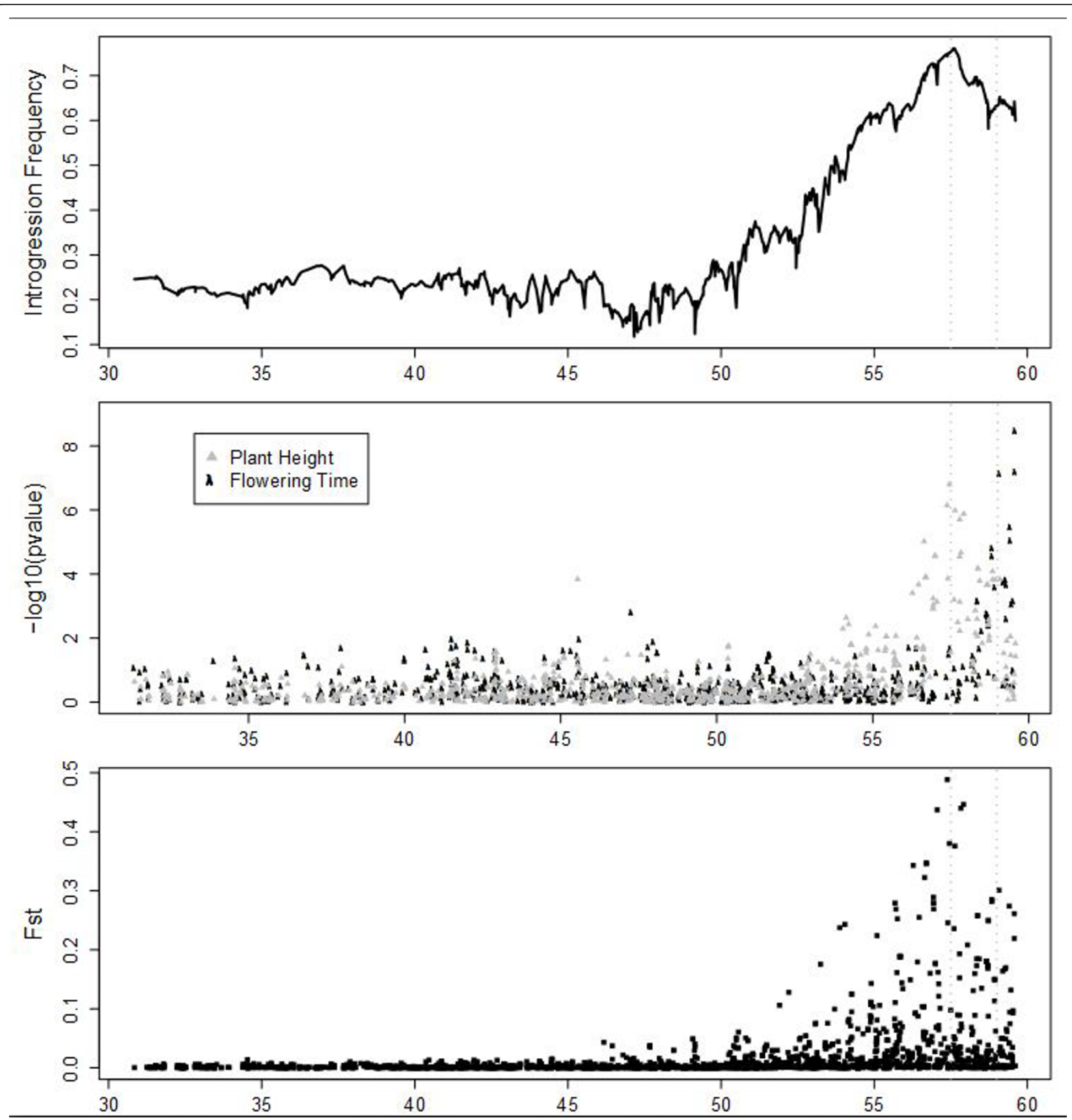

Figure 4 Introgression frequency, phenotypic associations, and population differentiation in the Dw 1 region on sorghum chromosome 9. Panels are the same as in Figures 2 and 3. The locations of putative QTL for plant height and maturity are shown with vertical dashed gray lines.

approaches - introgression mapping, association mapping, and population differentiation $\left(\mathrm{F}_{\mathrm{st}}\right)$ - to characterize the genetic architecture of adaptation to temperate-zone grain production in sorghum. Our novel introgression mapping strategy exploited recombination and selection previously imposed by plant breeders to map three major genomic regions, one of which no longer harbors functional variation in temperate-adapted SC lines. Association mapping confirmed that the $D w 1$ region contains separate QTL for plant height and flowering time. Significant differences in introgression frequency between subpopulations strongly suggest the existence of additional uncharacterized loci that affect plant height and flowering time in sorghum.

Linkage disequilibrium between at least four targeted loci on chromosome 6 has led to the introgression of a single elite haplotype across most of this chromosome in 


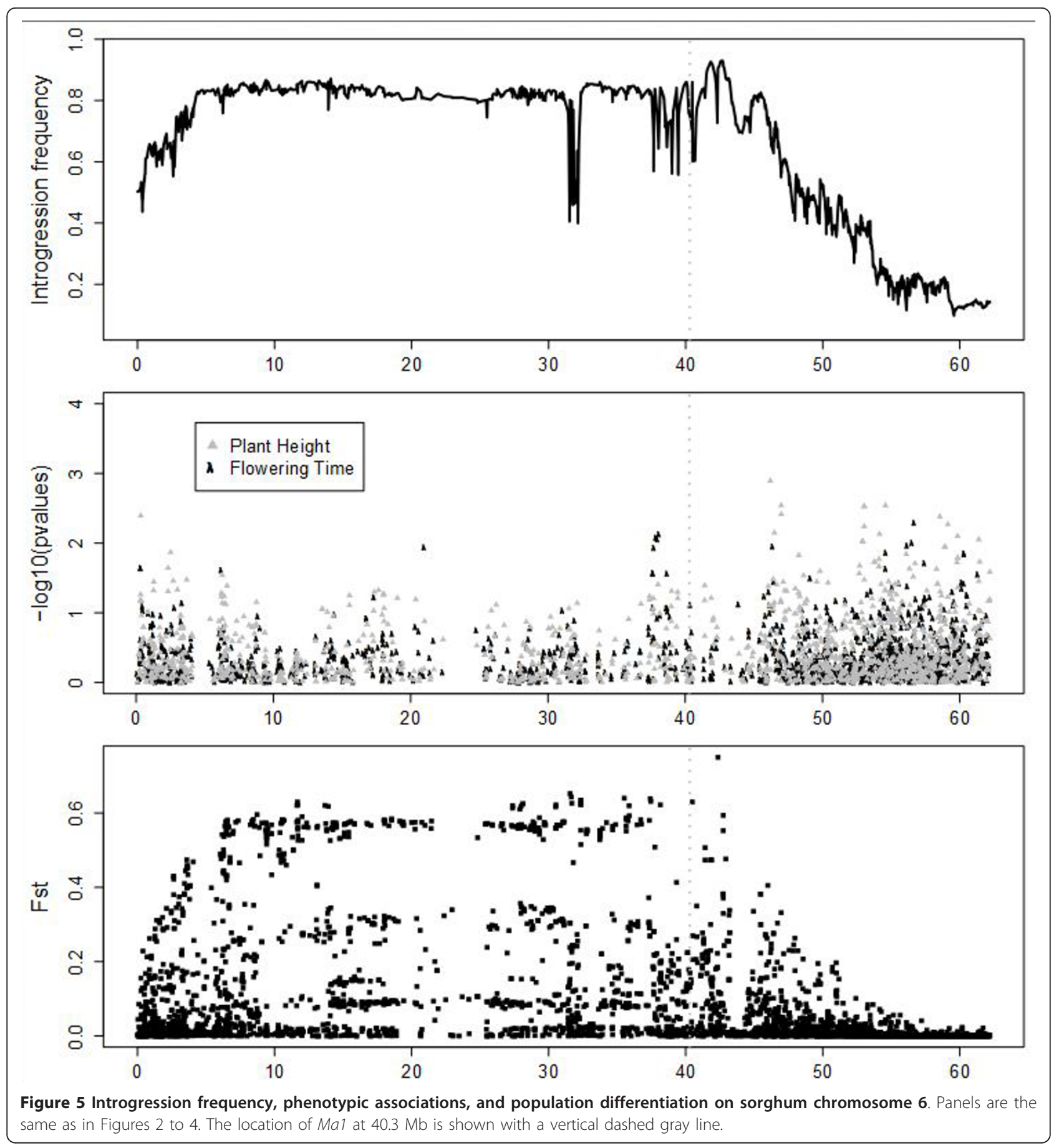

the majority of lines examined. Chromosome 6 contains roughly $10 \%$ of sorghum genes, for which very little functional diversity has been exploited for temperate sorghum breeding. This lack of diversity undoubtedly limits adaptive potential, especially for complex traits including resistance to abiotic and/or biotic stress. Increasing gene flow and recombination between tropical and temperate sorghum varieties and haplotypes will help unlock the genetic potential of this stress-tolerant crop to meet our rising demand for food, feed, and fuel in an era of increasing climatic volatility.

\section{Methods}

Plant materials, DNA extraction, and quantification

Seed for SC lines was obtained from the USDA-ARS Cropping Systems Research Laboratory (Lubbock, TX, 


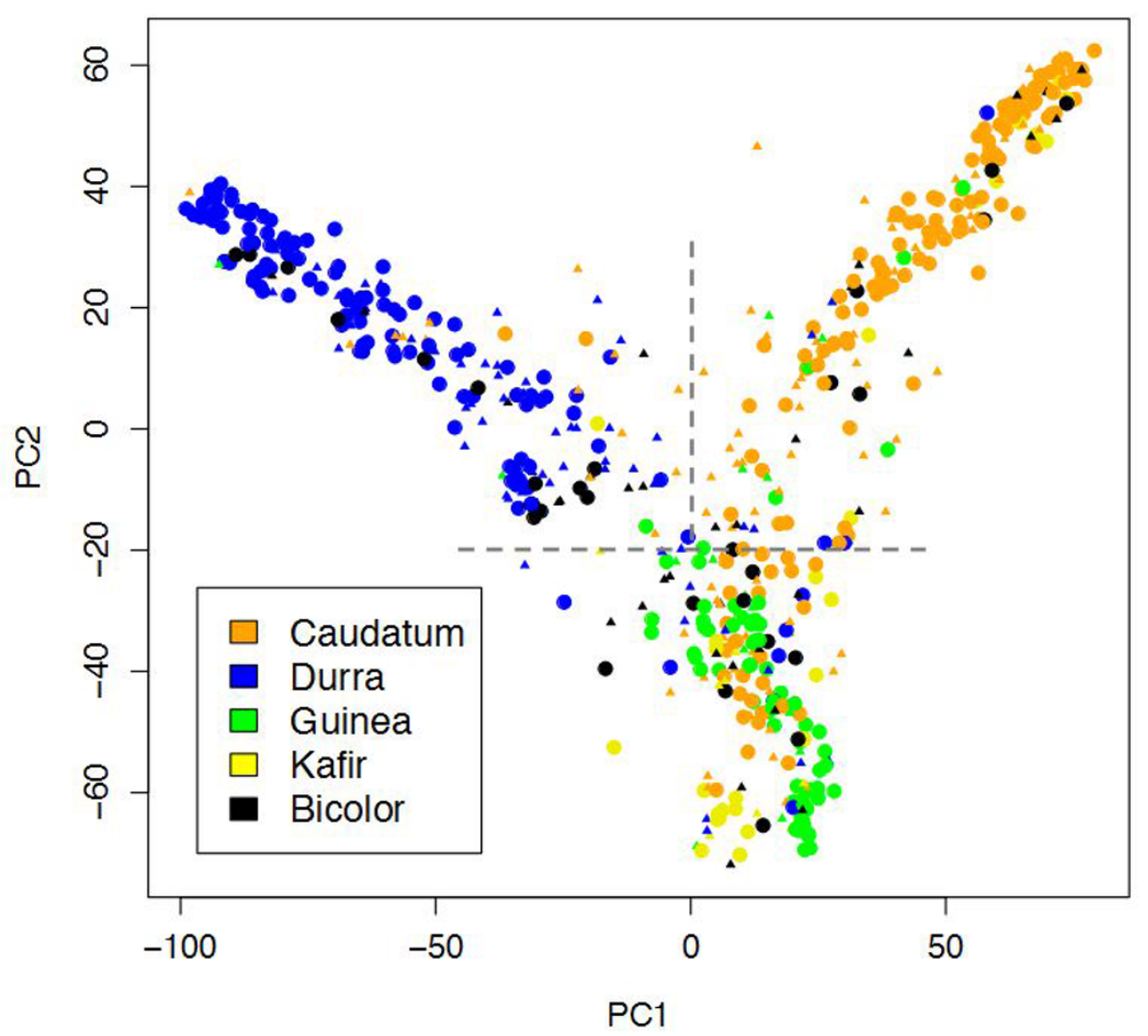

Figure 6 Sorghum racial identity and subpopulation structure. PCA plot of the 580 exotic progenitor (EP) lines genotyped in this study. Each dot represents an EP line, colored according to its morphologically-defined race. Larger circles and smaller triangles represent EP lines with more and fewer than $10 \%$ unexpected genotypes, respectively. The grey dashed lines indicate the criteria used to assign EP lines to genetic groups for subpopulation-specific introgression mapping.

USA) and seed for EP lines was obtained from the National Plant Germplasm System (NPGS [27]). Information on the geographic origins and morphological racial classification of each SC line were obtained from Texas A\&M University (Additional File 1). Three independent seed sources of the elite donor BTx406 were obtained from the NPGS (PI 656020), the USDA-Cropping Systems Research Laboratory, and Texas A\&M University. Genomic DNA was extracted from etiolated seedlings approximately 3 days after germination using a modified CTAB protocol [28] and quantified using PicoGreen (Invitrogen, NY, USA).

\section{SNP library creation}

Libraries were prepared using a protocol modified from Poland et al. 2012 [18]. Genomic DNA (approximately $250 \mathrm{ng}$ ) was double digested with either PstI-HF and BfaI or PstI-HF and HinP1I at $37^{\circ} \mathrm{C}$ for $2 \mathrm{~h}$ with heat inactivation at $80^{\circ} \mathrm{C}$ for $20 \mathrm{~min}$. Digested DNA was ligated to two separate adapters using T4 ligase with $1 \mathrm{mM}$ ATP. The first adapter contains the Illumina forward sequencing primer, one of 96 unique barcodes, and the PstI overhang. The second adapter contains the Illumina reverse sequencing primer and the overhang for either BfaI or HinP1I. The full list of adapters is shown in Additional File 11. Ligation reactions were held at $25^{\circ} \mathrm{C}$ for $2 \mathrm{~h}$ followed by heat inactivation at $65^{\circ} \mathrm{C}$ for $20 \mathrm{~min}$. Pooled DNA from 96 barcoded libraries was cleaned using a 2:1 ratio of AmpureXP Beads (Beckman Coulter, CA, USA) to DNA solution using a Magnetic Particle Concentrator (Invitrogen, NY, USA) with two washes in 95\% ethanol and resuspension in elution buffer (EB; $10 \mathrm{mM}$ Tris). Cleaned DNA pools were amplified using Illumina primers in a 2X PhusionHF Master Mix (New England Biolabs, MA, USA) with cycler conditions as follows: $98^{\circ} \mathrm{C}$ $30 \mathrm{~s}, 15$ cycles $\left(98^{\circ} \mathrm{C} 10 \mathrm{~s}, 68^{\circ} \mathrm{C} 30 \mathrm{~s}, 72^{\circ} \mathrm{C} 30 \mathrm{~s}\right), 72^{\circ} \mathrm{C}$ $5 \mathrm{~min}$. Samples were run on agarose gels to confirm the presence of a genomic smear and cleaned a second time with AMPure beads. Amplified DNA sizes and relative concentrations were assessed using an Agilent Bioanalyzer 2100 and Agilent DNA1000 Kit (Agilent Technologies Inc., CA, USA) and PicoGreen. The two separately digested samples were combined in equimolar concentrations and diluted to $10 \mathrm{nM}$ in library buffer (EB + 0.05\% 
Tween-20) and submitted to the W.M. Keck Center at the University of Illinois for single-end sequencing on the Illumina HiSeq2000. The Keck Center performed an additional qPCR assay on each library to adjust concentrations before sequencing.

\section{Genotype data analysis}

SNPs were called from Illumina fastq files using the TASSEL GBS pipeline [20]. Only 64 bp tags present at least 10 times in the dataset were considered. Alignment was performed using BWA [29] with the default settings. Inbred lines and SNPs with $>95 \%$ missing data were discarded. SNPs were not filtered by minor allele frequency, as rare SNPs are especially useful for inferring introgression events between pairs of lines (Figure 1B). Heterozygous genotypes accounted for $0.3 \%$ of the total dataset. Partial imputation using the TASSEL GBS pipeline reduced the proportion of missing data from approximately $66 \%$ to approximately $20 \%$. For the association and $\mathrm{F}_{\mathrm{st}}$ analyses, the remaining missing data were imputed using BEAGLE. This yielded substantially fewer unexpected genotypes than direct imputation using BEAGLE without prior partial imputation (data not shown).

\section{Mapping unanchored contigs in the sorghum genome}

We defined a set of 213 SNPs from 31 unanchored contigs that had at least 20 introgression calls and an introgression frequency of at least $10 \%$, and calculated linkage disequilibrium $\left(\mathrm{r}^{2}\right)$ between introgression scores in the 213 unanchored SNPs and our complete set of 46,062 SNPs with introgression scores in the 390 SC-EP pairs that were placed on the sorghum physical map (V1.0 [30]). Most (181) of the unanchored SNPs mapped uniquely to a single chromosome, with a mean of 8.4 mapped SNPs tied for the highest $\mathrm{r}^{2}$ across a mean physical distance of 9.1 Mb (Additional File 6).

\section{Calculation of introgression scores and frequencies}

For each SNP, an introgression was scored as either present (1), when a genotype was shared between the SC line and the donor line, or absent (0), when a genotype was shared between the SC line and its EP line. Missing data for presence/ absence of introgressions were inferred as the mean of each flanking marker weighted by its physical distance (Figure 1D). Missing data proximal and distal to the first and last informative markers on a chromosome, respectively, were assigned the value of the closest informative marker. Once missing data were imputed, introgression frequencies were calculated at each SNP as the percentage of SC lines with an introgression.

\section{Subpopulation assignment and permutations}

Principal component analysis (PCA) was performed in EP lines in R [31] using the $\operatorname{prcomp}()$ function and a dataset of 22,203 SNPs with minor allele frequencies $>10 \%$ in the set of 1,160 SC and EP lines (580 pairs). EP lines were assigned to subpopulations using values for $\mathrm{PC} 1$ and $\mathrm{PC} 2$ as follows: (1) lines with $\mathrm{PC} 2<-20$ were assigned to the guinea/kafir (GK) group; (2) lines with PC2 >-20 and PC1 $>0$ were assigned to the caudatum (C) group; (3) lines with PC2 $>-20$ and PC1 $<0$ were assigned to the durra (D) group. Introgressed regions excluded from the analysis in Additional File 8 were defined as locations $<55 \mathrm{Mb}$ on chromosome $6,>50 \mathrm{Mb}$ on chromosome 7 , and $>50 \mathrm{Mb}$ on chromosome 9 . Significance of subpopulation differences in introgression frequency was assessed by randomly assigning SC lines to subpopulations of equivalent size $(137,131$, and 122 individuals) and calculating introgression frequencies across the three permuted subpopulations. For each permutation, the maximum range of introgression frequencies across the three subpopulations was recorded for each chromosome. Two hundred permutations were performed and $\alpha$ was set to 0.05 .

\section{Phenotypic data and association mapping}

The 580 genotyped SC lines were grown in $6 \mathrm{~m}$ plots with $0.76 \mathrm{~m}$ row spacing in Urbana, IL in the summers of 2011 and 2012 and phenotyped for plant height and flowering time. Plant height was measured as the distance $(\mathrm{cm})$ from the ground to the penultimate or 'preflag' leaf on one representative plant per row. Flowering time was measured as the time (days from planting) at which $50 \%$ of the plants in the row had initiated anthesis. Phenotypic data from each year were normalized and the mean normalized value across all years was used for association mapping. The GAPIT package in $\mathrm{R}$ [32] was used to conduct marker-trait associations using the default parameters. Markers included all SNPs discovered in this study with minor allele frequencies $\geq 10 \%$. Missing SNP data were imputed using BEAGLE.

\section{Data availability}

Raw genotyping-by-sequencing read data have been deposited in the Sequenced Read Archive [SRA: SRP022956]. Introgression scores have been included in a table as Additional File 12.

\section{Additional material}

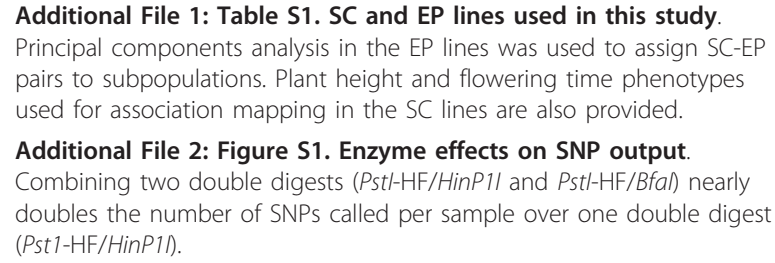


Additional File 3: Figure S2. Principal Component Analysis (PCA) of BTx406 seed source libraries. Twenty-eight libraries were created for BTX406 seed from three different sources (GRIN, Cornell, and Lubbock). The three outlier libraries from the GRIN collection were removed due to low concordance.

Additional File 4: Figure S3. Introgression maps for 390 SC lines. Additional File 5: Table S2. Number and percentage of introgressed, unexpected, and informative markers for each SC-EP pair. Additional File 6: Table S3. Physical map positions of unanchored SNPs

Additional File 7: Figure S4. Introgression frequency, phenotypic associations, and population differentiation in the Ma1-DW2 region on sorghum chromosome 6. Panels are the same as in Figures 3 to 6 . The locations of Ma1 at $40.3 \mathrm{Mb}$ is shown with a vertical dashed gray line.

Additional File 8: Figure S5. PCA of SC lines with and without SNPs in the three major introgressed regions.

Additional File 9: Figure S6. Subpopulation-specific introgression frequencies.

Additional File 10: Table S4. Phenotypic associations with plant height and flowering time in 580 SC lines.

Additional File 11: Table S5. List of barcoded adapters used in library preparation.

Additional File 12: Table S6. Raw introgression scores.

\section{Abbreviations}

EP: exotic progenitor; GBS: genotyping-by-sequencing; SC: sorghum conversion; SNP: single nucleotide polymorphism.

\section{Competing interests}

The authors declare that they have no competing interests.

\section{Authors' contributions}

PB conceived the project idea. $\mathrm{CT}, \mathrm{JM}, \mathrm{RH}$, and PB performed data collection and analysis. $\mathrm{PB}$ and $\mathrm{CT}$ wrote the manuscript.

\section{Acknowledgements}

This project was supported by startup funding from the Energy Biosciences Institute to PB. Charlie Woodfin (USDA-ARS Lubbock, retired) and Bill Rooney (TAMU) provided seed. We also wish to acknowledge the stellar technical support provided by Alvaro Hernandez and staff at the University of Illinois' Keck Center.

\section{Authors' details}

${ }^{1}$ Energy Biosciences Institute, University of Illinois, Urbana, IL, USA.

${ }^{2}$ Department of Crop Sciences, University of Illinois, Urbana, IL, USA.

Received: 28 March 2013 Revised: 11 June 2013

Accepted: 26 June 2013 Published: 26 June 2013

\section{References}

1. Gepts P: Crop domestication as a long-term selection experiment. Plant Breeding Reviews 2004, 24:1-44.

2. Harlan JR, De Wet JMJ, Price EG: Comparative evolution of cereals. Evolution 1973, 27:311-325.

3. Purugganan MD, Fuller DQ: The nature of selection during plant domestication. Nature 2009, 457:843-848.

4. Khush GS: Green revolution: preparing for the 21 st century. Genome 1999, 42:646-655.

5. Peng J, Richards DE, Hartley NM, Murphy GP, Devos KM, Flintham JE, Beales J, Fish LJ, Worland AJ, Pelica F: "Green revolution" genes encode mutant gibberellin response modulators. Nature 1999 400:256-260.

6. Ashikari M, Sasaki A, Ueguchi-Tanaka M, Itoh H, Nishimura A, Datta S, Ishiyama K, Saito T, Kobayashi M, Khush GS: Loss-of-function of a rice gibberellin biosynthetic gene, GA20 oxidase (GA20ox-2), led to the rice 'green revolution'. Breeding Science 2002, 52:143-150.

7. Hedden P: The genes of the Green Revolution. Trends Genet 2003, 19:5-9

8. Food and Agriculture Organization of the United Nations. [http://www. fao.org/index_en.htm].

9. Smith CW, Frederiksen RA: Sorghum: Origin, History, Technology, and Production Hoboken, NJ: John Wiley \& Sons; 2000

10. Quinby JR: Sorghum improvement and the genetics of growth College Station, TX: Texas A\&M University Press; 1974.

11. Murphy RL, Klein RR, Morishige DT, Brady JA, Rooney WL, Miller FR, Dugas DV, Klein PE, Mullet JE: Coincident light and clock regulation of pseudoresponse regulator protein 37 (PRR37) controls photoperiodic flowering in sorghum. Proc Natl Acad Sci USA 2011, 108:16469-16474.

12. Childs KL, Miller FR, Cordonnier-Pratt MM, Pratt LH, Morgan PW, Mullet JE: The Sorghum Photoperiod Sensitivity Gene, Ma3, Encodes a Phytochrome B. Plant Physiol 1997, 113:611-619.

13. Multani DS, Briggs SP, Chamberlin MA, Blakeslee JJ, Murphy AS, Johal GS: Loss of an MDR Transporter in Compact Stalks of Maize br2 and Sorghum dw3 Mutants. Science 2003, 302:81-84.

14. Klein RR, Mullet JE, Jordan DR, Miller FR, Rooney WL, Menz MA, Franks CD, Klein PE: The effect of tropical sorghum conversion and inbred development on genome diversity as revealed by high-resolution genotyping. Crop Science 2008, 48:S-12.

15. Morris GP, Ramu P, Deshpande SP, Hash CT, Shah T, Upadhyaya HD, RieraLizarazu O, Brown PJ, Acharya CB, Mitchell SE, Harriman J, Glaubitz JC, Buckler ES, Kresovich S: Population genomic and genome-wide association studies of agroclimatic traits in sorghum. Proc Natl Acad Sci USA 2013, 110:453-458

16. Brown PJ, Rooney WL, Franks C, Kresovich S: Efficient mapping of plant height quantitative trait loci in a sorghum association population with introgressed dwarfing genes. Genetics 2008, 180:629-637.

17. Stephens JC, Miller FR, Rosenow DT: Conversion of alien sorghums to early combine genotypes. Crop Science 1967, 7:396-396.

18. Poland JA, Brown PJ, Sorrells ME, Jannink J-L: Development of highdensity genetic maps for barley and wheat using a novel two-enzyme genotyping-by-sequencing approach. PLOS ONE 2012, 7:e32253.

19. Elshire RJ, Glaubitz JC, Sun Q, Poland JA, Kawamoto K, Buckler ES, Mitchell SE: A robust, simple genotyping-by-sequencing (GBS) approach for high diversity species. PLOS ONE 2011, 6:e19379.

20. Buckler Lab for Maize Genetics and Diversity. [http://www.maizegenetics. net/].

21. Hill WG: Variation in genetic identity within kinships. Heredity 1993, 71:652-653.

22. Mace ES, Jordan DR: Location of major effect genes in sorghum (Sorghum bicolor (L.) Moench). Theor Appl Genet 2010, 121:1339-1356.

23. Parh DK, Jordan DR, Aitken EA, Mace ES, Jun-Ai P, Mclntyre CL, Godwin ID: QTL analysis of ergot resistance in sorghum. Theor App/ Genet 2008, 117:369-382.

24. Klein RR, Rodriguez-Herrera R, Schlueter JA, Klein PE, Yu ZH, Rooney WL: Identification of genomic regions that affect grain-mould incidence and other traits of agronomic importance in sorghum. Theor Appl Genet 2001, 102:307-319.

25. Satish K, Srinivas G, Madhusudhana R, Padmaja PG, Nagaraja Reddy R, Murali Mohan S, Seetharama N: Identification of quantitative trait loci for resistance to shoot fly in sorghum [Sorghum bicolor (L.) Moench]. Theor Appl Genet 2009, 119:1425-1439.

26. Lin Z, Li X, Shannon LM, Yeh C-T, Wang ML, Bai G, Peng Z, Li J, Trick HN, Clemente TE, Doebley J, Schnable PS, Tuinstra MR, Tesso TT, White F, Yu J: Parallel domestication of the Shattering1 genes in cereals. Nat Genet 2012, 44:720-724.

27. National Plant Germplasm System. [http://www.ars-grin.gov/npgs/]

28. Mace ES, Buhariwalla KK, Buhariwalla HK, Crouch JH: A high-throughput DNA extraction protocol for tropical molecular breeding programs. Plant Mol Biol Rep 2003, 21:459-460.

29. Li H, Durbin R: Fast and accurate short read alignment with BurrowsWheeler transform. Bioinformatics 2009, 25:1754-1760.

30. Paterson AH, Bowers JE, Bruggmann R, Dubchak I, Grimwood J, Gundlach H, Haberer G, Hellsten U, Mitros T, Poliakov A: The Sorghum bicolor genome and the diversification of grasses. Nature 2009, 457:551-556.

31. The Comprehensive R Archive Network. [http://cran.r-project.org/]. 
32. Lipka AE, Tian F, Wang Q, Peiffer J, Li M, Bradbury PJ, Gore MA, Buckler ES, Zhang Z: GAPIT: genome association and prediction integrated tool. Bioinformatics 2012, 28:2397-2399.

doi:10.1186/gb-2013-14-6-r68

Cite this article as: Thurber et al:: Retrospective genomic analysis of sorghum adaptation to temperate-zone grain production. Genome Biology 2013 14:R68.

Submit your next manuscript to BioMed Central and take full advantage of:

- Convenient online submission

- Thorough peer review

- No space constraints or color figure charges

- Immediate publication on acceptance

- Inclusion in PubMed, CAS, Scopus and Google Scholar

- Research which is freely available for redistribution 